\title{
A genome-wide association study of seed protein and oil content in soybean
}

\author{
Eun-Young Hwang ${ }^{1}$, Qijian Song ${ }^{2}$, Gaofeng Jia ${ }^{2}$, James E Specht ${ }^{3}$, David L Hyten ${ }^{2,4}$, Jose Costa ${ }^{1,5}$ and Perry B Cregan ${ }^{2^{*}}$
}

\begin{abstract}
Background: Association analysis is an alternative to conventional family-based methods to detect the location of gene(s) or quantitative trait loci (QTL) and provides relatively high resolution in terms of defining the genome position of a gene or QTL. Seed protein and oil concentration are quantitative traits which are determined by the interaction among many genes with small to moderate genetic effects and their interaction with the environment. In this study, a genome-wide association study (GWAS) was performed to identify quantitative trait loci (QTL) controlling seed protein and oil concentration in 298 soybean germplasm accessions exhibiting a wide range of seed protein and oil content.
\end{abstract}

Results: A total of 55,159 single nucleotide polymorphisms (SNPs) were genotyped using various methods including Illumina Infinium and GoldenGate assays and 31,954 markers with minor allele frequency $>0.10$ were used to estimate linkage disequilibrium (LD) in heterochromatic and euchromatic regions. In euchromatic regions, the mean $L D\left(r^{2}\right)$ rapidly declined to 0.2 within $360 \mathrm{Kbp}$, whereas the mean LD declined to 0.2 at 9,600 Kbp in heterochromatic regions. The GWAS results identified 40 SNPs in 17 different genomic regions significantly associated with seed protein. Of these, the five SNPs with the highest associations and seven adjacent SNPs were located in the 27.6-30.0 Mbp region of Gm20. A major seed protein QTL has been previously mapped to the same location and potential candidate genes have recently been identified in this region. The GWAS results also detected 25 SNPs in 13 different genomic regions associated with seed oil. Of these markers, seven SNPs had a significant association with both protein and oil.

Conclusions: This research indicated that GWAS not only identified most of the previously reported QTL controlling seed protein and oil, but also resulted in narrower genomic regions than the regions reported as containing these QTL. The narrower GWAS-defined genome regions will allow more precise marker-assisted allele selection and will expedite positional cloning of the causal gene(s).

Keywords: GWAS, Glycine max, Seed protein and oil content, Single nucleotide polymorphism, Linkage disequilibrium

\section{Background}

Association studies provide an alternative to conventional family-based methods for detecting the genomic location of genes or quantitative trait loci (QTL). While both rely on the correlation between DNA marker alleles and the phenotypic expression of a trait of interest, association studies can provide relatively higher resolution in terms of defining the genomic position of a gene or QTL, since it can be applied to naturally occurring populations such as human populations or germplasm collections.

\footnotetext{
* Correspondence: perry.cregan@ars.usda.gov

${ }^{2}$ USDA, Agricultural Research Service, Soybean Genomics and Improvement Lab, Beltsville, MD 20705, USA

Full list of author information is available at the end of the article
}

The important difference between the degree of QTL detection possible with these natural populations and that possible with progeny derived from crosses between two individuals is the level of linkage disequilibrium (LD).

LD is the non-random association of alleles at different loci in a population. The detection of genes or QTL depends on the level of LD between a causal mutation and physically linked markers. The higher the degree of association between marker alleles and the phenotypic variants, the greater the likelihood that the phenotypic causal mutation is physically linked to the marker. A confounding factor that influences the success of association study is the presence of population structure. 
Population structure is the result of allele frequency differences between different populations that arise as a result of population history. Factors such as selection, migration, local adaptation, geographical isolation, or genetic drift can result in population structure. It is well known that population structure can cause spurious associations between markers and the trait under study [1], because of allele frequency differences between subpopulations in a population, rather than genuine genetic associations with the trait of interest.

Owing to the lack of sufficient numbers of DNA markers, early association studies in plant species used the candidate gene approach to identify specific single nucleotide polymorphisms (SNPs) or genes controlling a phenotypic trait of interest such as, flowering time [2], endosperm color [3] and kernel starch biosynthesis [4] in maize (Zea mays ssp. mays), enzymatic discoloration in potato (Solanum tuberosum L.) [5], flowering time in sorghum (Sorghum bicolor L. Moench) [6], and aluminum tolerance in triticale (X Triticosecale Wittmack) [7]. However, the availability of high throughput DNA sequencing and genotyping technologies has provided a platform for conducting genome-wide association studies (GWAS). Such studies have verified the location of loci associated with frost tolerance in barley (Hordeum vulgare L.) [8]; leaf architecture [9]; southern leaf blight [10], and waterlogging tolerance [11] in maize (Zea mays L.); agronomic traits in rice (Oryza sativa L.) [12]; flowering time [13] and defense metabolites [14] in Arabidopsis (Arabidopsis thaliana L. Heynh) and plant architecture and flowering time in sunflower [15]. In soybean (Glycine $\max$ L. Merr.), a GWAS was performed to detect genes/markers associated with iron deficiency chlorosis [16] and chlorophyll and chlorophyll fluorescence parameters [17].

Seed protein and oil content in soybean are quantitatively inherited traits determined by the interaction of a number of genes subject to genotype $\times$ environment interactions. Many seed protein and oil QTL have been reported in a number of studies over the past two decades (SoyBase, the USDA, ARS Soybean Genetics and Genomics Database). These QTL, identified using linkage analysis of populations derived from crosses of two parents with contrasting seed protein and oil concentration, have been detected in many different genomic regions throughout all 20 chromosomes. Several of these QTL have been identified three or more times at identical or very similar chromosomal positions in different populations, which suggests that these QTL are not likely false positives. Because these regions likely contain a gene or genes with relatively large genetic effects on seed protein and oil content, their reidentification in a GWAS targeted at seed protein and oil would provide a measure of the success of GWAS in an independent test of its ability to detect the presence of seed protein and oil QTL.

In the research reported here, the degree of LD was assessed in 298 soybean germplasm accessions obtained from the USDA Soybean Germplasm Collection. Seed protein and oil concentration were measured in seeds harvested from plants grown in replicated field trials at two locations. The resulting seed composition data were used to evaluate the GWAS approach, and ultimately to provide an assessment of the likely success of GWAS when it is used to detect the QTL controlling the two well-known soybean quantitative traits-seed protein and oil content.

\section{Results}

Linkage disequilibrium in euchromatic and heterochromatic genome regions

A total of 31,954 SNPs distributed across the soybean genome with minor allele frequency $>0.10$ and missing data of less than $25 \%$ was used for the estimation of LD level in the 298 soybean germplasm accessions. These SNP markers spanned $950.1 \mathrm{Mbp}$, which represents approximately $86.4 \%$ of the $1.1 \mathrm{Gbp}$ soybean genome, resulting in an average SNP density of 1 SNP every 17.0 Kbp in euchromatic regions and 1 SNP every 100.1 Kbp in heterochromatic regions. In euchromatic regions, the mean level of LD measured by $D^{\prime}$ declined very rapidly to 0.5 within $600 \mathrm{Kbp}$, and mean LD as measured by $r^{2}$ dropped sharply to 0.2 at $360 \mathrm{Kbp}$ (Figure 1). However, in heterochromatic regions, the mean value of $D^{\prime}$ remained greater than 0.5 until about $15,000 \mathrm{Kbp}$ and mean LD as measured by $r^{2}$ did not decline to 0.2 until about 9,600 Kbp (Figure 1).

\section{Population structure}

A total of 42,368 SNPs positioned on the 20 soybean chromosomes was used to estimate the most likely number $(K)$ of subgroups in the 298 germplasm accessions. The most likely $K$ value was $K=17$. The accessions within seven subgroups originated from a single Asian country and the other nine subgroups were comprised of a mix of accessions from different countries. In terms of maturity group, eight subgroups consisted of accessions with the same maturity group, whereas the other eight subgroups were a mix of accessions of different maturity groups.

\section{Seed protein and oil phenotypes}

The germplasm accessions were selected based upon data in the USDA Germplasm Resources Information Network (GRIN) database (http://www.ars-grin.gov/), to represent two distinct groups-one group with normal seed protein content ( 40 to $43 \%$ ) and the other with high seed protein content (46 to 51\%). However, seed protein 


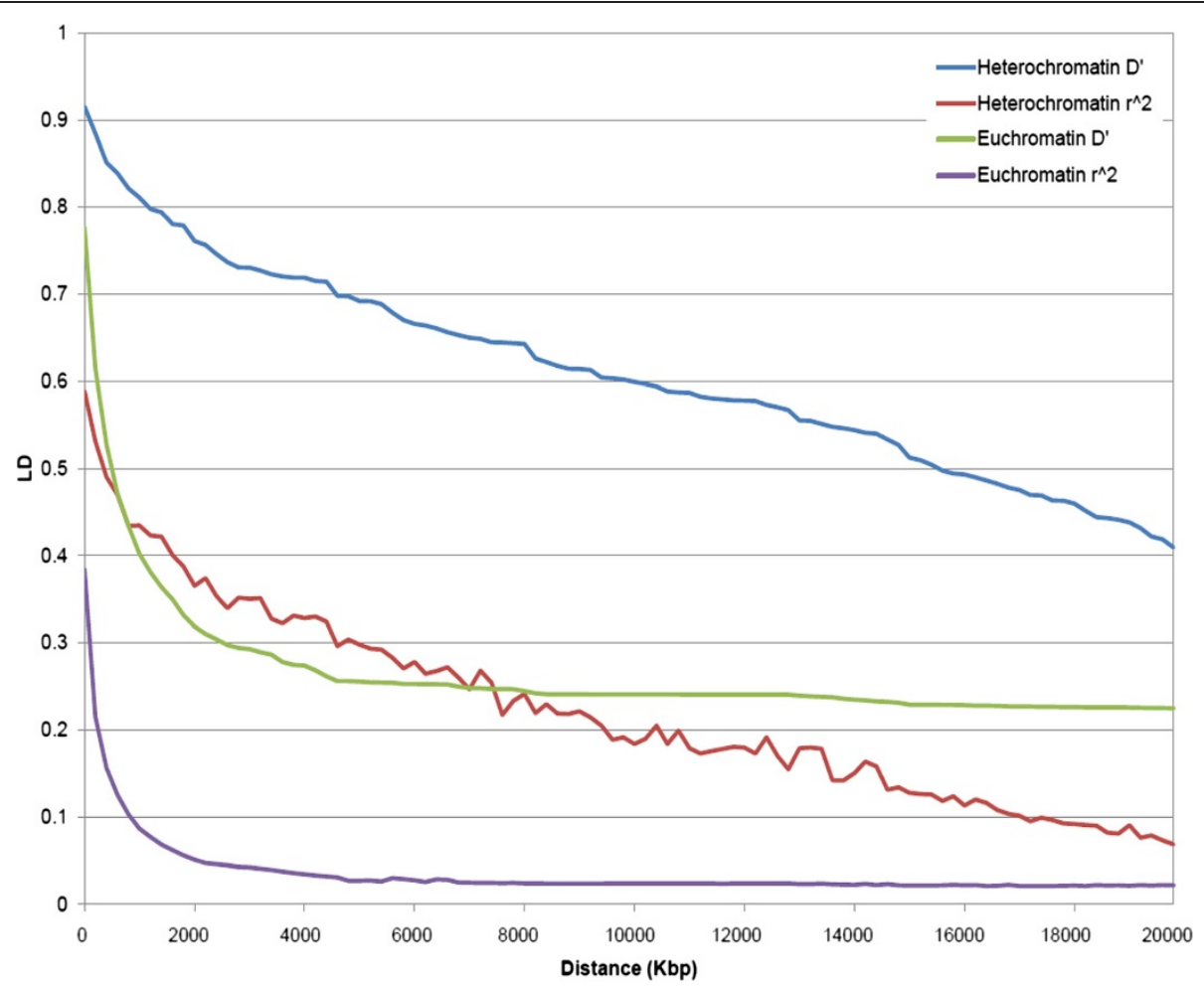

Figure 1 The mean level of LD in heterochromatic and euchromatic chromosome regions. The mean LD was estimated using all pairs of loci located within $20 \mathrm{Mbp}$ of physical distance. The $X$-axis indicates the distance between marker pairs and the $Y$-axis indicates LD level. The green and purple lines respectively denote mean $D^{\prime}$ and mean $r^{2}$ in euchromatic regions, and the blue and red lines respectively denote mean $D^{\prime}$ and mean $r^{2}$ in heterochromatic regions.

content measured in seeds grown in replicated hill-plot trials at Beltsville, MD and Lincoln, NE showed continuous variation with a range from 35 to $50 \%$ (Figure $2 \mathrm{~A}$ ). Experimental error was low in each trial, given the coefficient of variation $(\mathrm{CV})$ values of $5.77 \%$ in the $\mathrm{MD}$ experiment and $5.99 \%$ in the NE experiment, with an overall $\mathrm{CV}$ of $5.67 \%$ in the 2-location analysis. The analysis of variance indicated that the accessions differed significantly $(\mathrm{P}<0.0001)$ in seed protein content, and no significant interaction between accessions and locations was detected (Table 1). The correlation coefficient ( $r$ ) of seed protein concentration between the $\mathrm{MD}$ and $\mathrm{NE}$ experiments was quite high, $\mathrm{r}=0.98(\mathrm{P}<0.0001)$. The correlation of the accession mean seed protein measured in the NE and MD experiments with the GRIN accession protein value was moderate, $r=0.62(\mathrm{P}<0.0001)$ and $r=0.61 \quad(P<0.0001)$, respectively. The seed oil content of the accessions displayed a pattern similar to the seed protein content relative to the GRIN oil values (Figure 2B). The analysis of variance for seed oil revealed that accessions were significantly different $(\mathrm{P}<0.0001)$ and as was the case with protein, there was no significant accession $\times$ location interaction for oil (Table 2). The correlation of accession seed oil content between the two locations was high, $r=0.95(\mathrm{P}<0.0001)$, but was only moderately high, $\mathrm{r}=0.77(\mathrm{P}<0.0001)$ and $\mathrm{r}=0.78$ $(\mathrm{P}<0.0001)$, between the GRIN accession values and those measured at the NE and MD locations, respectively. The correlation between mean seed protein and oil contents in the MD experiment and in the NE experiment were $r=-0.64$ and $r=-0.66$, respectively. The heritability of seed protein and seed oil concentrations was $77.9 \%$ and $78.33 \%$, respectively. Such heritability values are higher than would be typical in breeding studies.

\section{Genome-wide association study for the genes controlling seed protein and oil concentration}

A GWAS was performed with the mixed linear model (MLM) which greatly reduced false positive rates as shown in quantile-quantile plots (Additional file 1). The results of the genome-wide association study for seed protein content are presented in Figure 3A. A significant association $(-\log \mathrm{P}>3)$ with seed protein concentration was observed for 40 SNPs located in 17 different genomic regions in 10 of the 20 chromosomes (Additional file 2). Nine of the 17 regions had more than two markers with significant association and the physical distance between markers ranged from 1,786 to 1,723,908 bp. More than a quarter (i.e., 12) of these 40 SNPs had physical positions 

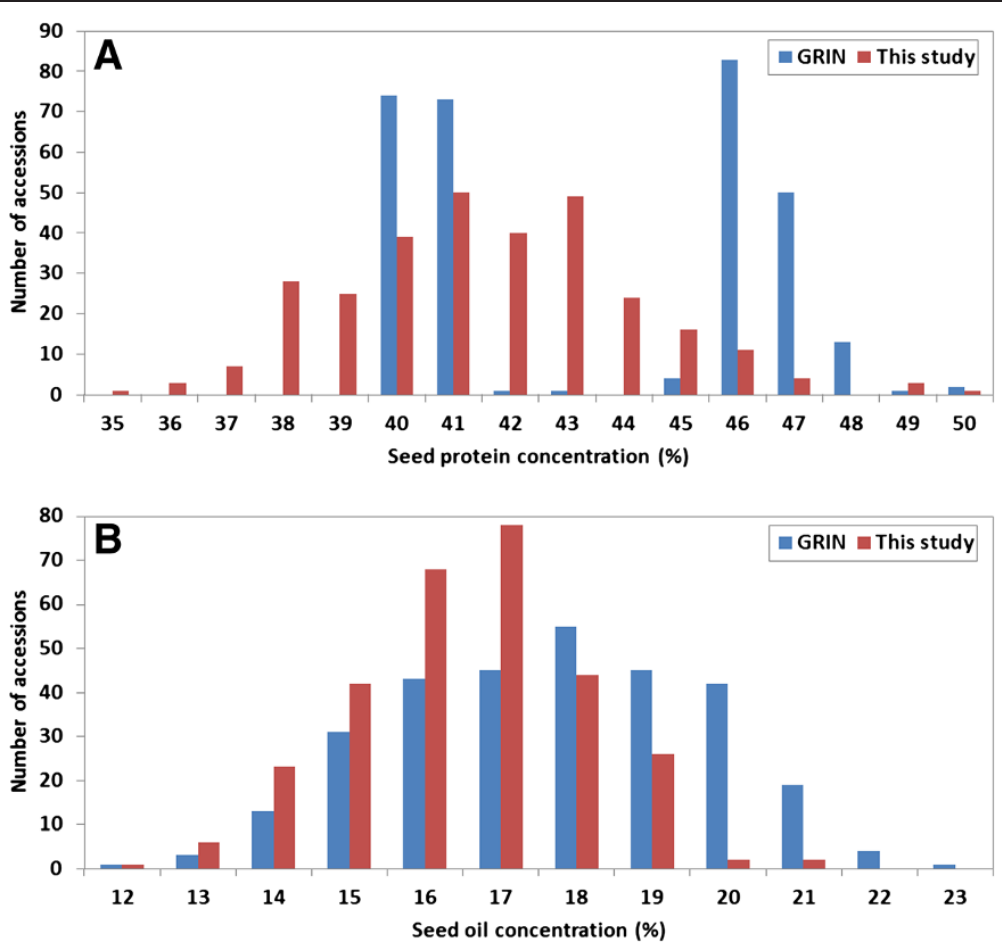

Figure 2 Seed protein and oil concentration in the GRIN database vs. that determined in this study. Seed protein (A) and seed oil (B) concentrations of the soybean germplasm accessions, respectively, reported in the GRIN database vs. the percentage determined in this study from seed harvested from two-replicate trials conducted at two locations (Beltsville, MD; and Lincoln, NE) in 2003. Blue bars are data from the GRIN database and red bars are data from this study.

in the 27.9-30.0 Mbp segment of Gm20, all of which were in complete LD as indicated by $D^{\prime}$ (Figure 4 ). Of the 40 SNPs, five SNPs in the 27.9-30.0 Mbp segment of Gm20 exhibited the highest association with seed protein content. This is not surprising given the fact that this is a heterochromatic region with very extensive $\mathrm{LD}$, while the 28 other markers with significant association to protein content were located in euchromatic regions where the mean level of LD declines very rapidly. A significant association with seed oil content was detected for 25 SNPs, and these were physically located in 13 regions on 12 of the 20 chromosomes (Figure $3 \mathrm{~B}$ and Additional file 3) where seven regions included more than two markers with the distance between markers ranging from 5,108 to 470,370 bp. All of these markers were

Table 1 Analysis of variance of seed protein content

\begin{tabular}{cccccc}
\hline Source & DF & SS & MS & F value & Pr $>$ F \\
\hline Accession & 297 & 6874.21 & 23.15 & 77.6 & $<0.0001$ \\
Location & 1 & 6.95 & 6.95 & 23.2 & 0.0389 \\
Accession $\times$ location & 297 & 99.06 & 0.3 & 0.185 & 1 \\
Replications (locations) & 2 & 4.59 & 2.29 & 1.413 & 0.2442 \\
Error & 518 & 840.5 & 1.62 & & \\
\hline
\end{tabular}

Analysis of variance of harvested seed of G. max accessions from plants grown in 2003 in replicated hill plot trials conducted in fields located in Beltsville, MD and near Lincoln, NE. located in euchromatic regions, except the four markers located on Gm20. The SNP showing the highest association with oil (i.e., a- $\log \mathrm{P}$ value of 4.71) was located at the $4.92 \mathrm{Mbp}$ position on Gm09. There were seven markers from three regions on $\mathrm{Gm} 08, \mathrm{Gm} 09$, and $\mathrm{Gm} 20$ associated with both protein and oil seed content (Table 3). At six of these seven marker loci there was a negative relationship between the protein effect versus that on oil i.e., the allele associated with increased protein content was always associated with decreased oil content or vice versa. The aforementioned SNP at the $4.92 \mathrm{Mbp}$ position on Gm09 was the exception-one allele was associated with increased protein and oil content and the alternative allele with both lower protein and oil content.

Table 2 Analysis of variance of seed oil content

\begin{tabular}{cccccc}
\hline Source & DF & SS & MS & F value & Pr $>$ F \\
\hline Accession & 290 & 2414.49 & 8.33 & 14.88 & $<0.0001$ \\
Location & 1 & 0.69 & 0.69 & 1.23 & 0.3667 \\
Accession $\times$ location & 290 & 66.06 & 0.23 & 0.411 & 1 \\
Replications (locations) & 2 & 3.49 & 1.74 & 3.12 & 0.045 \\
Error & 520 & 291 & 0.56 & & \\
\hline
\end{tabular}

Analysis of variance of harvested seed of G. max accessions from plants grown in 2003 in replicated hill plot trials conducted in fields located in Beltsville, MD and near Lincoln, NE. 


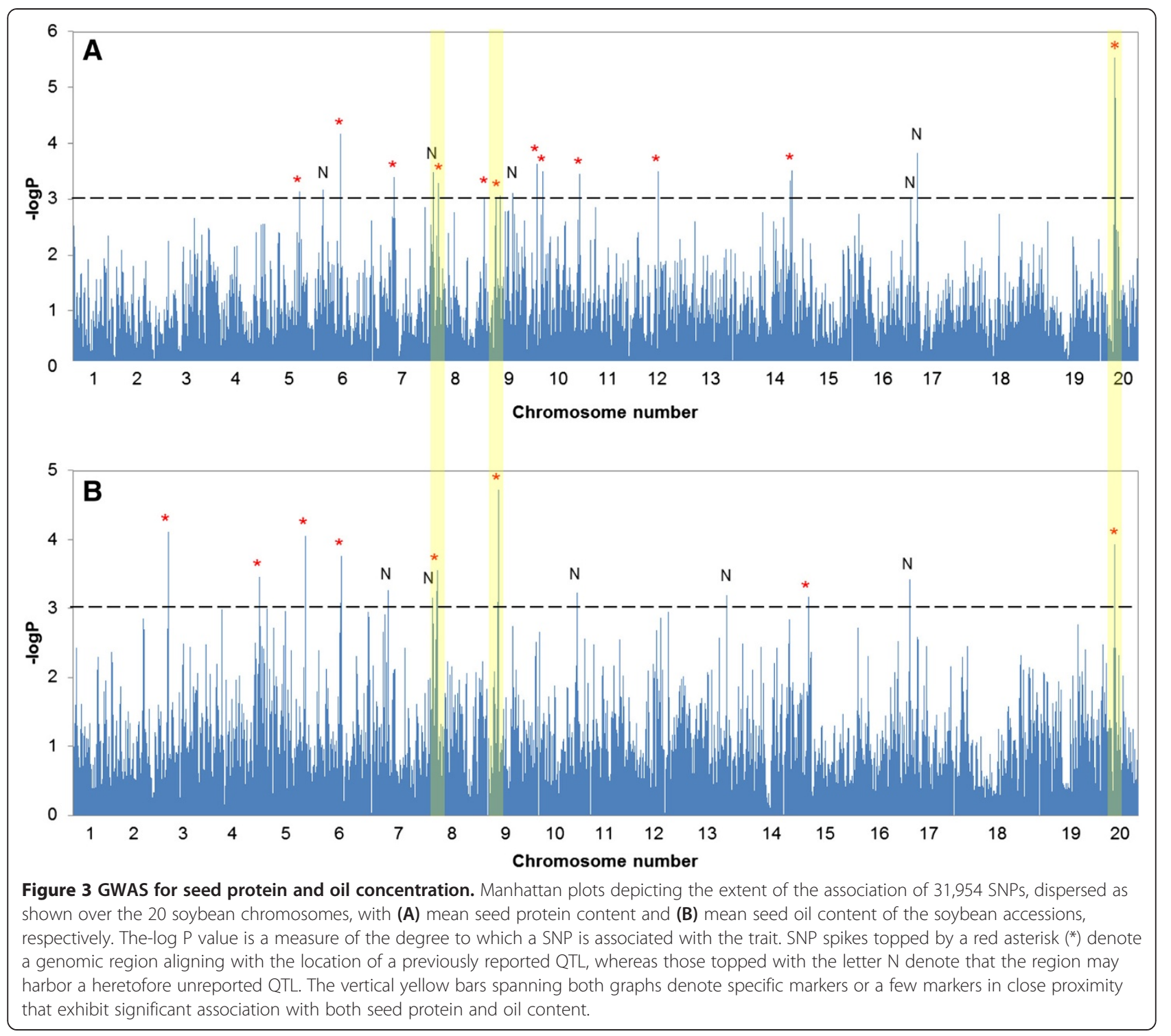

\section{Discussion}

Soybean germplasm accessions used in this study that include landraces collected in China, Korea, and Japan over a 60 year period represent an excellent reservoir of genetic variation for the application of a GWAS. It is assumed that these accessions possess a diversity of alleles. Such diversity can be examined (and possibly exploited) for soybean genetic improvement by identifying alleles that would be useful in achieving that genetic improvement. GWAS offers one approach for accomplishing that objective.

\section{Linkage disequilibrium}

An important consideration in the application of genome-wide association for gene discovery is the extent of LD. Because of obvious differences in the extent of LD between different chromosomal regions, we elected to define LD separately for the euchromatic and heterochromatic regions of the 20 soybean chromosomes. The reliability of the approach used in the present study, i.e., to define the heterochromatic region of each chromosome as the region between the two inflection points of the cumulative genetic distance plotted against the cumulative physical distance, was previously examined in rice. In that species, the heterochromatic regions defined by suppressed recombination rate was in agreement with that identified by the conventional DAPI staining method [18]. The plot of genetic distance on physical distance clearly indicated a higher recombination per physical distance in euchromatic regions $v s$. heterochromatic regions. Approximately five times greater recombination per unit of physical distance occurs in soybean euchromatic versus heterochromatic regions. In the grass species, sorghum (Sorghum bicolor (L.) Moench), 


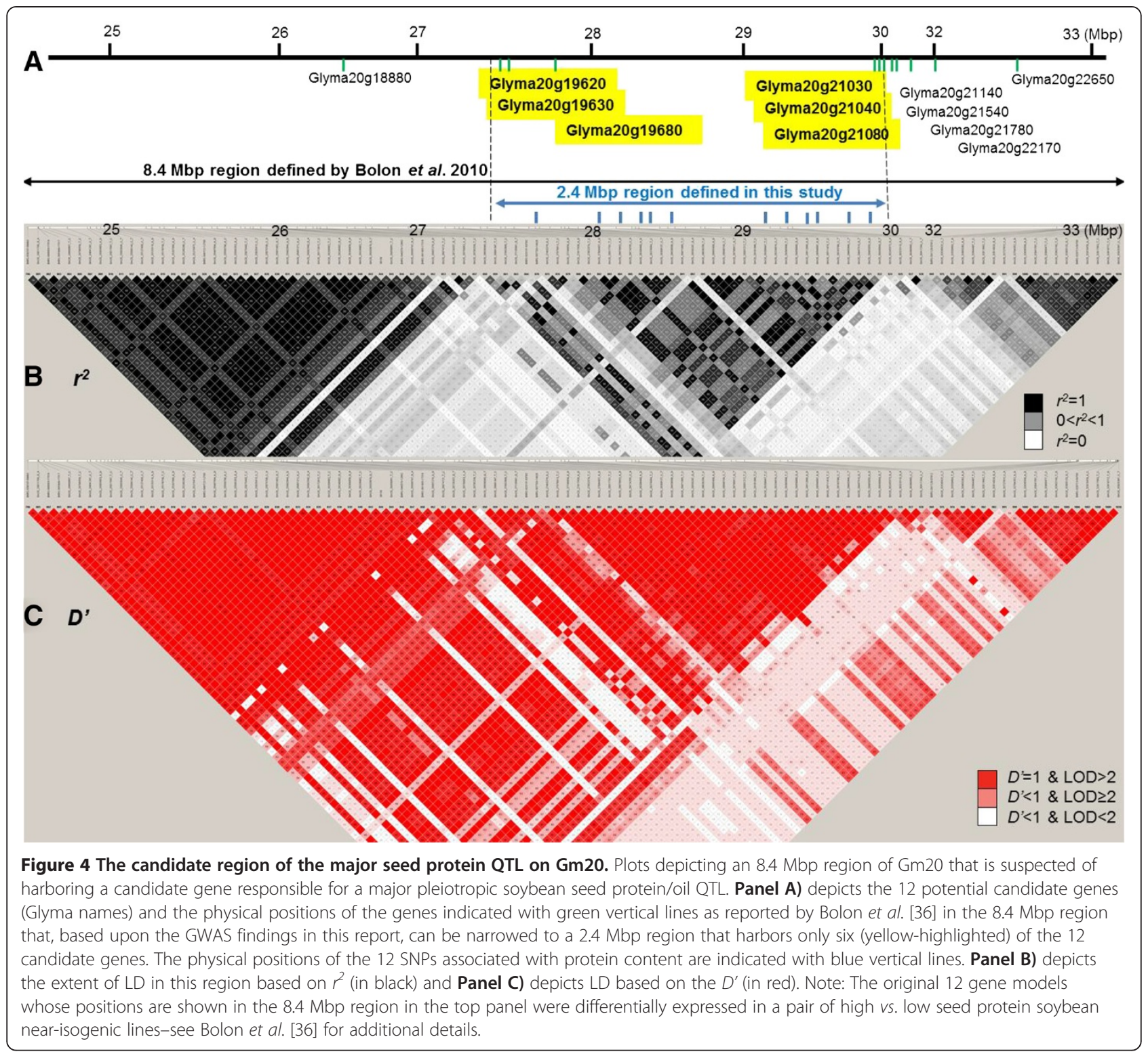

the recombination rate in euchromatic regions was approximately 34 times higher than that in heterochromatic regions [19]. In fact, $97-98 \%$ of the recombination occurred in the euchromatin of this species [20]. The difference in recombination rate between the heterochromatic regions and the euchromatic regions explains the difference in the extent of LD observed in the two chromosomal regions. The different levels of LD as indicated by $r^{2}$ between different species and different chromosome regions within species can be explained by a combination of population histories including mating system, mutation rate, founding effects, the magnitude of selection, admixture, and genetic drift. The large difference in the LD level in euchromatic and heterochromatic regions, as shown in the present study, supports our contention that the density of markers required for GWAS should be adjusted to take into account the changing ratio, along the length of a given chromosome, of recombination to genetic distance. In euchromatic regions with high recombination to physical distance, a high marker density will be required, whereas in heterochromatic regions, a lower marker density is acceptable. Of course, until a whole genome sequence and a densely populated genetic map become available, it is not possible to precisely define the ratio of genetic to physical distance across the genome of a species to which a GWAS is being conducted.

\section{Association analysis for seed protein and oil content}

The challenge for association studies in crop plants is the identification of genes associated with quantitatively inherited agriculturally important traits. Currently, many seed protein and oil QTL have been reported at many 
Table 3 SNP loci associated with both seed protein and oil content

\begin{tabular}{|c|c|c|c|c|c|c|c|c|c|c|c|c|c|c|}
\hline \multirow[t]{2}{*}{ Chr } & \multirow[t]{2}{*}{ SNP ID } & \multicolumn{2}{|c|}{$-\log P$} & \multicolumn{4}{|c|}{$\begin{array}{l}\text { Mean protein content (\%) } \\
\text { associated with SNP allele }\end{array}$} & \multicolumn{4}{|c|}{$\begin{array}{c}\text { Mean oil content (\%) } \\
\text { associated with SNP allele } \\
\end{array}$} & \multicolumn{2}{|c|}{ Difference } & \multirow{2}{*}{$\begin{array}{c}\text { Protein } \\
\text { vs. oil } \\
\text { relationship }\end{array}$} \\
\hline & & Protein & Oil & G & $A$ & $\mathrm{~T}$ & C & G & $A$ & $\mathrm{~T}$ & $\mathrm{C}$ & Protein & Oil & \\
\hline 8 & BARC_1.01_Gm08_9683120_C_T & 3.22 & 3.25 & & & 42.35 & 40.08 & & & 16.78 & 18.35 & 2.27 & 1.57 & Negative \\
\hline 8 & BARC_1.01_Gm08_9741542_T_C & 3.28 & 3.55 & & & 39.95 & 42.37 & & & 18.43 & 16.76 & 2.42 & 1.66 & Negative \\
\hline 9 & BARC_1.01_Gm09_4921120_C_T & 3.06 & 4.71 & & & 41.61 & 42.15 & & & 16.41 & 17.06 & 0.55 & 0.65 & Positive \\
\hline 20 & BARC_1.01_Gm20_29395999_T_C & 5.52 & 3.93 & & & 41.46 & 44.32 & & & 17.34 & 15.59 & 2.86 & 1.75 & Negative \\
\hline 20 & BARC_1.01_Gm20_29512680_A_G & 4.96 & 3.69 & 44.22 & 41.46 & & & 15.65 & 17.34 & & & 2.76 & 1.69 & Negative \\
\hline 20 & BARC_1.01_Gm20_29594697_A_G & 5.52 & 3.93 & 44.32 & 41.46 & & & 15.59 & 17.34 & & & 2.86 & 1.75 & Negative \\
\hline 20 & BARC_1.01_Gm20_29983050_A_G & 4.82 & 3.03 & 44.32 & 41.50 & & & 15.65 & 17.30 & & & 2.82 & 1.65 & Negative \\
\hline
\end{tabular}

The mean protein and oil content of the genotypes with the alternative SNP alleles, the difference in protein and oil content between the genotypes with the alternative alleles and the direction (negative or positive) of the protein vs. oil effects associated with the alleles at each locus.

${ }^{a} \mathrm{~A}$ negative relationship indicates that the allele associated with greater protein content was associated with reduced oil content and vice versa. A positive relationship indicates that the allele associated with greater protein content was also associated with greater oil content and the alternative allele was associated with both reduced protein and oil content.

positions across the 20 soybean chromosomes/linkage groups in numerous studies (SoyBase, www.soybase.org). The genome positions where QTL were reported a number of times in studies using different sources of high seed protein and oil germplasm should be good candidates for the validation of associations detected via GWAS. All the previously reported seed protein and oil QTL listed in SoyBase were identified using linkage analysis and thus, the causal gene(s) could be located a substantial distance (in $\mathrm{cM}$ ) from the markers which were reported to be genetically linked to the QTL. Therefore, the exact position of the QTL determined by linkage analysis could not be precisely determined. However, with the release of Version 4.0 of the soybean genetic map [21], which was produced from a JoinMap 3.0 analysis of five soybean mapping populations that collectively segregated for over 5,000 markers, it is possible to more narrowly define the regions to which previously reported protein and oil QTL had been mapped. Using this information, it is possible with some confidence to relate the physical position of the reported QTL from different studies with the SNP positions identified by GWAS in the present study. In that regard, we were able to align the 17 genome regions associated with seed protein identified in this study with previously reported seed protein QTL locations, and based on that alignment, determined that QTL had previously been reported in 12 of the 17 regions (Figure $3 \mathrm{~A}$ and Additional file 2). Notably, the seed protein QTL on Gm20 has been reported seven times, the QTL at the 14.7 Mbp position on $\mathrm{Gm} 06$ six times, the regions located on $\mathrm{Gm} 05, \mathrm{Gm} 07$, and $\mathrm{Gm} 15$ four times, the region on Gm09 three times, and the regions at $43.9 \mathrm{Mbp}$ on $\mathrm{Gm} 08,1.4 \mathrm{Kbp}$ on $\mathrm{Gm} 10$, and on $\mathrm{Gm} 12$ have been reported in two previous studies. Of the 13 regions associated with seed oil content, the regions on Gm05, Gm06, and Gm20 had been previously reported more than three times and the regions on $\mathrm{Gm} 02$ and $\mathrm{Gm} 04$, the region at $9.9 \mathrm{Mbp}$ on $\mathrm{Gm} 09$, and the region on Gm15 have been reported twice. In addition, there were QTL that have been reported in previous studies which could not be found in this study. One reason could be that since the accessions used in this study were from the maturity groups II, III and IV, QTL identified in accessions from other maturity groups are not present in the germplasm accessions used in this study. For example, we did not find QTL reported in studies using very early or late maturity groups such as three QTL identified in populations derived from maturity group $00 \times 00$ [22], three QTL from maturity group $0 \times 0[23,24]$, nine QTL from maturity group VI $\times$ VI [25], and three QTL from maturity group $0 \times \mathrm{I}[26,27]$. A second reason may be that many protein and oil QTL have been identified in a number of different populations, however, if a QTL is controlled by a rare allele present only in a specific accession used in creating a QTL mapping population, it could not be detected in a GWAS such as is reported here. The inability of GWAS to detect rare alleles occurring in one or a few members of a population under study is well documented [28,29].

The main differences between the findings of an association study versus linkage analysis are firstly, the ability to detect a range of genes controlling the phenotype under study rather than just those segregating in a given mating of two parents and secondly, the increased resolution resulting from historical recombination rather than the limited recombination in the progeny of a biparental population. The QTL identified on Gm20 provides an example of the increased resolution of GWAS. This seed protein QTL has been reported seven times in different G. soja $\times$ G. $\max$ and G. $\max \times$ G. $\max$ populations with $R^{2}$ values ranging from 0.15 to 0.65 [22,30-35]. This QTL on Gm20 has been recognized as a candidate region for possibly cloning the causal gene(s) controlling seed protein content. Bolon et al. [36] analyzed 
gene expression of developing seeds in a pair of near isogenic lines (NILs) contrasting in seed protein concentration and differing for the region of Gm20 containing this major seed protein QTL. Differential transcript accumulation in the developing seeds of the NILs was detected for 12 candidate genes. The region defined by Bolon et al. [36] in which these genes reside spans approximately 8.4 Mbp from 24.5 to $32.9 \mathrm{Mbp}$ on Gm20. Based on the results of the LD estimation in the current study, we verified that the region defined by Bolon et al. [36] possessed two large LD blocks, as determined by $r^{2}$, each spanning regions of approximately $3 \mathrm{Mbp}$, one from 24.5 to 27.6 Mbp and the other from 27.6 to $30.0 \mathrm{Mbp}$ (Figure 4). All the SNPs showing significant association with seed protein concentration were located in the 27.6 to $30.0 \mathrm{Mbp}$ region. Thus, our GWAS results support a narrowing of the candidate gene region of this major seed protein QTL on Gm20 to about 2.4 Mbp versus the previously defined region of 8.4 Mbp [36]. Only six of the 12 genes identified by Bolon et al. [36] were located within the GWASdefined 2.4 Mbp region (Figure 4). The gene candidates that remain include Hsp22.5 (Glyma20g19680), a putative ammonium transporter AMT1 (Glyma20g21030), an ATP synthase D chain (Glyma20g21080) (Figure 4) and three genes with limited similarity to known genes. We can assume that one or more of these genes may likely be the causative gene(s) associated with soybean seed protein content. This high resolution mapping demonstrates the capacity of GWAS to utilize historical recombination to increase resolution [37].

The GWAS for seed oil concentration also successfully defined 13 chromosome regions with high resolution, of which eight regions corresponded with previously reported oil QTL. Thus, in the case of both seed protein and oil content, five chromosome regions were identified at which no previously reported QTL had been detected. These regions may be of particular interest to soybean breeders and geneticists as sources of genetic variation to alter soybean seed protein and oils levels.

It has been recognized that there is a negative correlation between seed size and seed protein concentration $[32,34]$, but more importantly, an even stronger negative correlation between seed oil and seed protein content [22,38-41]. G. soja has an extremely small seed size and much higher seed protein concentration $(>50 \%)[42,43]$ than G. max. The average seed protein content in most G. $\max$ germplasm accessions is approximately $42.1 \%$ [44]. The reasonable explanation could be that selection had been imposed for larger seed types during domestication by ancient soybean farmers which had a concurrent genetic consequence of lower seed protein concentration. The lower seed protein concentration resulting from selection would also simultaneously lead to the increase in seed oil concentration. The possible cause for this would be either very tight genetic linkage of the genes controlling seed protein and oil concentration or that both traits are controlled by the same gene(s) [30]. The latter is more likely, given that high seed protein and the low seed oil almost always co-segregate and given the fact that many attempts to separate low seed oil from high seed protein (or the inverse) have been unsuccessful [30,32]. The results of GWAS from the current study were not in complete agreement with this assertion given results for the SNP at the $4.92 \mathrm{Mbp}$ position on Gm09 at which one allele was associated with both higher protein and oil content and the alternative allele with lower protein and oil content. This is a QTL that should be of interest to those breeding for seed constituents.

One suggested virtue of association studies is the ability to take advantage of existing phenotypic data. However, there are also obstacles to performing association studies using existing phenotypic data, especially in the case of quantitative traits such as soybean seed protein and oil content. The seed protein and oil data obtained from GRIN were derived from field evaluations of the USDA Soybean Germplasm Collection conducted over the past 50 years at various locations and the evaluations included accessions from one or more maturity groups. The seed protein and oil data for the accessions used in the current study were not from a single field trail or set of trials in which all of the 298 accessions were included. Rather, the data were from a number of different trials grown in different environments and years. A quantitative trait defined in different studies and determined in a range of different environments and years is likely to produce different accession values that are confounded by these non-genetic effects, and it is, of course, the accession means that are used in GWAS. Such may be the case of the seed protein and oil concentration from the GRIN database used in this study (Figure 2A and B). There was only a correlation of $r=0.61$ for seed protein concentration and 0.78 for seed oil concentration between the replicated field data from the current study $v s$. the data reported in the GRIN. We have a high level of confidence in the data obtained in the current study given the low coefficient of variation in the two replicated field experiments at two locations and the high correlation of the seed protein and oil values between the two locations. Thus, it is at least a possibility that the advantage often suggested for association study, i.e., the availability of large amounts of existing phenotypic data may, in some cases, not be the advantage that is often assumed.

\section{Conclusions}

In this study, we performed a GWAS to detect genome regions controlling the quantitative traits, seed protein and oil content, using 42,368 SNP markers in a genetically 
diverse set of the 298 soybean germplasm accessions. Despite the relatively low level of $\mathrm{LD}$ and complex population structure, we were able to successfully identify many of the previously reported QTL associated with soybean seed protein and oil, and we also were able to further narrow the size of the genome region in which those QTL were likely to be located. The chromosome regions defined in this study can be used for further analysis to identify the causal gene(s) as well as to identify DNA markers that can be used in selection to alter soybean seed protein and oil in a predictable manner. The SNP data used in this study will soon be available through SoyBase (http:// soybase.org/) for more than 19,000 G. max and G. soja germplasm accessions in the USDA Soybean Germplasm Collection based upon the analysis of the entire USDA Soybean Germplasm Collection with the Illumina Infinium SoySNP50K BeadChip described by Song et al. [45]. These genotypic data should be a useful resource for the detection of agriculturally important genes/QTL using association analysis.

\section{Methods}

\section{Plant materials}

Soybean germplasm accessions were selected based upon seed protein content (\% on a dry weight basis) as reported in the GRIN (Germplasm Resources Information Network, U.S. Department of Agriculture, Agricultural Research Service, http://www.ars-grin.gov/npgs/index.html) database (Additional file 4). A total of 298 accessions were identified so as to create two groups-a case group including 151 accessions with high seed protein values ranging from 46 to $51 \%$, and a control group including 147 accessions with more typical seed protein values ranging from 40 to $43 \%$. The selection of accessions for the two groups was constrained to ensure balance between the groups in terms of geographical origin in the Far East (China, Korea, Japan), maturity group (II, III \& IV), and other known phenotypic and morphological traits. Such balancing was done in an attempt to mitigate differences in population structure between the two groups. Genomic DNA of all accessions was extracted from bulked young leaf tissue grown in the greenhouse using the CTAB method [46].

\section{Field trials and measurement of protein and oil in the harvested seed}

Field tests were conducted using a randomized complete block design with two replicates of hillplots grown at Beltsville, MD and two replicates grown at Lincoln, NE in 2003. For an analysis of total seed nitrogen, the seeds were dried for one day at $60^{\circ} \mathrm{C}$ and ground with a coffee grinder. The powdered grain was stored in an air-tight polyethylene bag and weighed in small tin capsules (LECO, St. Joseph, MI) to a targeted powder weight of approximately $0.2 \mathrm{~g}$. The percentage of total nitrogen in the grain powder was determined using a LECO CHN 2000 analyzer (LECO, St. Joseph, MI) [47]. The seed protein percentage was calculated by multiplying the total nitrogen percentage by 6.25 . The seed oil percentage was determined with approximately $10 \mathrm{~g}$ of seed using a Maran pulsed NMR (Resonance Instruments, Witney, Oxfordshire, UK), followed by the field induction decay-spin echo procedure [48]. Protein and oil concentrations were expressed on a\% dry weight basis.

\section{Genotyping}

A total of 55,159 single nucleotide polymorphisms (SNPs) were genotyped in the 298 accessions, with 52,041 analyzed using an Illumina Infinium assay [45] and 3,072 SNPs analyzed using the Illumina GoldenGate assay following the protocol described by Fan et al. [49] and Hyten et al. [50]. In addition, there were 46 SNP markers genotyped by direct sequencing and a singlebase-extension method using the Luminex flow cytometer as described by Choi et al. [51]. A total of 1,363 SNPs were analyzed using both Illumina GoldenGate and Illumina Infinium assays. After eliminating redundant SNPs, non-polymorphic SNPs and SNPs with $>25 \%$ missing data, a total of 42,368 SNPs (Additional file 5) remained. The physical positions of these markers in the soybean genome were determined using the whole genome assembly of Williams 82 soybean (Glyma 1.01) at the U.S. Department of Energy, Joint Genome Institute, Walnut Creek, CA (http://www.phytozome.net/soybean).

\section{Linkage disequilibrium estimation}

For the estimation of the level of LD, a total of 31,954 loci with minor allele frequency $>0.10$ and the number of missing data points less than $25 \%$ was used. Heterozygous alleles were treated as missing data. Only physically linked markers located within $20 \mathrm{Mbp}$ distances were used for LD estimation. Haploview 4.2 [52] was used to make all pair-wise comparisons of the alleles to calculate $r^{2}$ (the squared allele frequency correlation between two loci), and to compute $D^{\prime}$ (standardized disequilibrium coefficient) [53]. In addition, LD ( $D^{\prime}$ and $\left.r^{2}\right)$ was estimated separately for euchromatic and heterochromatic regions. The euchromatin and the heterochromatin regions of each of the 20 chromosomes were determined as follows: The physical positions of 3,321 SNP and 862 simple sequence repeat (SSR) markers mapped in the soybean genome $[21,51,54-56]$ were determined by BLAST analysis of the SNP and SSR-containing source sequences to the soybean whole genome sequence using the standalone Megablast software as previously described [57]. The cumulative genetic distances (cM) [21] were plotted against their cumulative physical distance (Mbp) to determine the base pair/centiMorgan relationship via the 
common SSR and SNP loci positions on the genetic linkage map and their physical position in the genome sequence along each chromosome. The region between the two inflection points of the cumulative genetic distance against cumulative physical distance on the plot was defined as the heterochromatin and the regions on each chromosome flanking the inflection points were defined as the euchromatin [45]. In order to provide an assessment of the difference in the extent of LD between euchromatic and heterochromatic regions, LD was calculated by a pairwise comparison of physically linked heterochromatic SNPs, and then two separate LD calculations were made for all pairs of markers from the two flanking euchromatic regions on each chromosome. The mean value of $\mathrm{LD}$ was estimated by calculating the mean LD of SNP pairs at distances of 0-200 Kbp, 200-400 Kbp, etc. to 19,800-20,000 Kbp in euchromatic and heterochromatic regions.

\section{Statistical analysis}

An analysis of variance was conducted to obtain the variance components which were used to calculate the heritability of seed protein and oil content. The variances of location, replications within locations, accessions, and the accession $\times$ location interaction were determined using the PROC GLM procedure of the Statistical Analysis System (SAS institute, Inc., Cary, NC). Genetic and environmental variances were extracted from the variance component estimates based on the expected mean squares. For the estimation of the heritability of seed protein and seed oil concentration, replications and locations were considered to be random effects.

The heritability of seed protein and oil concentration was defined as

$$
h_{B}^{2}=\frac{\sigma_{g}^{2}}{\sigma_{g}^{2}+\sigma_{e}^{2}},
$$

where $\sigma_{g}^{2}$ is the genetic variance among accessions, and $\sigma_{e}^{2}$ is the environmental variance which results from error and the accession $\times$ location interaction.

To obtain the matrix of population structure, a total of 42,368 SNPs were analyzed in the 298 germplasm accessions using the Admixture program v. 1.22 [58]. The 10-fold cross-validation procedure was performed with 25 random seeding replications for $K$ values from 2 to 30. The minimum mean standard error was when $K=17$. The kinship coefficient matrix that explained the most probable identity by state of each allele between individuals was estimated with the TASSEL program [59]. For a genome-wide association study, we compared the false positive rate using the general linear model (GLM), the mixed linear model (MLM), and the compressed MLM of the TASSEL program [59]. The MLM was as good as the compressed MLM and greatly reduced the false positive rate versus the GLM. For this study, the value of 0.001 was used as a Type I error significance threshold $\mathrm{P}$ value. As a verification of the genome regions identified in this study we compared the genomic locations of previously reported seed protein and oil QTL with the physical positions of the markers showing significant associations in this study.

\section{Additional files}

Additional file 1: Quantile-quantile plots for seed protein and oil content. Quantile-quantile plots of the general linear model (GLM) for seed protein (A) and oil (B), the mixed linear model (MLM) for seed protein (C) and oil (D), and the compressed MLM for seed protein (E) and oil (F).

Additional file 2: SNP markers associated with seed protein content QTL. Significantly associated markers (based on a $-\log P>3.0$ ) are numbered consecutively in the first column. The second column reports whether the QTL has been previously reported and the third column reports whether a marker(s) in the regions is also associated with seed oil content.

Additional file 3: SNP markers associated with seed oil content QTL. Significantly associated markers (based on a-logP > 3.0) are numbered consecutively in the first column. The second column reports whether the QTL has been previously reported and the third column reports whether a marker(s) in the region is also associated with seed protein content.

Additional file 4: Soybean germplasm accessions analyzed in this study. Soybean germplasm accessions [with Plant Introduction (PI) numbers] analyzed in this study and information including country of origin and phenotypic data reported in GRIN. The rightmost four columns display the seed protein and oil values reported in GRIN and those measured in the present study.

Additional file 5: The SNP allele present at 42,369 SNP loci analyzed in the $\mathbf{2 9 8}$ soybean germplasm accessions. Data includes the SNP Locus Name, the NCBI Assay ID (ss\#), the chromosome number (Chr) on which the SNP is located in the Williams 82 soybean whole genome sequence (Glyma 1.01 assembly, DOE, JGl http://www.phytozome. net/soybean) and the physical position in basepairs in the Glyma 1.01 assembly.

\section{Abbreviations}

GWAS: Genome-wide association study; SNPs: Single nucleotide polymorphisms; LD: Linkage disequilibrium; QTL: Quantitative trait locus; GRIN: Germplasm resources information network; SSR: Simple sequence repeat; CM: Centimorgan; CV: Coefficient of variation; K: Structure matrix; RFLP: Restriction fragment length polymorphism; NILs: Near isogenic lines; GLM: General linear model; MLM: And checks and the three experiments in mixed linear model.

\section{Competing interests}

The authors declare that they have no competing interests.

\section{Authors' contributions}

PBC designed and supervised the research; EYH analyzed the data, QS carried out the statistical analysis; GJ performed the Infinium assay; JES conducted the field trial to evaluate the seed protein and oil content; JC, EYH and PBC wrote the manuscript. All authors read and approved the manuscript.

\section{Acknowledgements}

We thank the National Science Foundation (IOS 0822258) and the United Soybean Board Project \#8265 for funding to support this work. 


\section{Author details}

${ }^{1}$ Department of Plant Science and Landscape Architecture, University of Maryland, College Park, MD 20742, USA. ${ }^{2}$ USDA, Agricultural Research Service, Soybean Genomics and Improvement Lab, Beltsville, MD 20705, USA. ${ }^{3}$ Agronomy \& Horticulture Department, University of Nebraska, Lincoln, NE 68583, USA. ${ }^{4}$ Present address: DuPont Pioneer, 8305 NW 62nd Ave., PO Box 7060, Johnston, IA 50131, USA. ${ }^{5}$ Present address: USDA-ARS, Crop Production and Protection, GWCC-BLTSVL, Beltsville, MD 20705, USA.

Received: 29 August 2013 Accepted: 21 December 2013

Published: 2 January 2014

\section{References}

1. Knowler WC, Williams RC, Pettitt DJ, Steinberg AG: Gm3;5,13,14 and type 2 diabetes mellitus: an association in American Indians with genetic admixture. Am J Hum Genet 1988, 43(4):520-526.

2. Thornsberry JM, Goodman MM, Doebley J, Kresovich S, Nielsen D, Buckler ES IV: Dwarf8 polymorphisms associate with variation in flowering time. Nat Genet 2001, 28(3):286-289.

3. Palaisa KA, Morgante M, Williams M, Rafalski A: Contrasting effects of selection on sequence diversity and linkage disequilibrium at two phytoene synthase loci. Plant Cell 2003, 15(8):1795-1806.

4. Wilson LM, Whitt SR, Ibanez AM, Rocheford TR, Goodman MM, Buckler ES IV: Dissection of maize kernel composition and starch production by candidate gene association. Plant Cell 2004, 16(10):2719-2733.

5. Urbany $C$, Stich B, Schmidt L, Simon L, Berding H, Junghans $H$, Niehoff KH, Braun A, Tacke E, Hofferbert HR, et al: Association genetics in Solanum tuberosum provides new insights into potato tuber bruising and enzymatic tissue discoloration. BMC Genomics 2011, 12:7-20.

6. Bhosale SU, Stich B, Rattunde HF, Weltzien E, Haussmann BI, Hash CT, Ramu $P$, Cuevas HE, Paterson AH, Melchinger AE, et al: Association analysis of photoperiodic flowering time genes in west and central African sorghum [Sorghum bicolor (L.) Moench]. BMC Plant Biol 2012, 12:32-41.

7. Niedziela A, Bednarek PT, Cichy H, Budzianowski G, Kilian A, Aniol A: Aluminum tolerance association mapping in triticale. BMC Genomics 2012, 13:67-82.

8. Visioni A, Tondelli A, Francia E, Pswarayi A, Malosetti M, Russell J, Thomas W, Waugh R, Pecchioni N, Romagosa I, et al: Genome-wide association mapping of frost tolerance in barley (Hordeum vulgare L.). BMC Genomics 2013, 14:424.

9. Tian F, Bradbury PJ, Brown PJ, Hung H, Sun Q, Flint-Garcia S, Rocheford TR McMullen MD, Holland JB, Buckler ES: Genome-wide association study of leaf architecture in the maize nested association mapping population. Nat Genet 2011, 43(2):159-162.

10. Kump KL, Bradbury PJ, Wisser RJ, Buckler ES, Belcher AR, Oropeza-Rosas MA, Zwonitzer JC, Kresovich S, McMullen MD, Ware D, et al: Genome-wide association study of quantitative resistance to southern leaf blight in the maize nested association mapping population. Nat Genet 2011, 43(2): 163-168

11. Zhang X, Tang B, Yu F, Li L, Wang M, Xue Y, Zhang Z, Yan J, Yue B, Zheng $Y$, et al: Identification of major QTL for waterlogging tolerance using genome-wide association and linkage mapping of maize seedlings Plant Mol Biol Rep 2013, 31:594-606.

12. Huang X, Wei X, Sang T, Zhao Q, Feng Q, Zhao Y, Li C, Zhu C, Lu T, Zhang $Z$, et al: Genome-wide association studies of 14 agronomic traits in rice landraces. Nat Genet 2010, 42(11):961-967.

13. Brachi B, Faure N, Horton M, Flahauw E, Vazquez A, Nordborg M, Bergelson J, Cuguen J, Roux F: Linkage and association mapping of Arabidopsis thaliana flowering time in nature. PLoS Genet 2010, 6(5):e1000940.

14. Chan EK, Rowe HC, Kliebenstein DJ: Understanding the evolution of defense metabolites in Arabidopsis thaliana using genome-wide association mapping. Genetics 2010, 185(3):991-1007.

15. Mandel JR, Nambeesan S, Bowers JE, Marek LF, Ebert D, Rieseberg LH, Knapp SJ, Burke JM: Association mapping and the genomic consequences of selection in sunflower. PLoS Genet 2013, 9(3):e1003378.

16. Mamidi S, Chikara S, Goos RJ, Hyten DL, Moghaddam SM, Cregan PB, McClean PE: Genome-wide association analysis identifies candidate genes associated with iron deficiency chlorosis in soybean. Plant Genome 2011, 4:154-164

17. Hao D, Chao M, Yin Z, Yu D: Genome-wide association analysis detecting significant single nucleotide polymorphisms for chlorophyll and chlorophyll fluorescence parameters in soybean (Glycine max) landraces. Euphytica 2012, 186:919-931.

18. Zhao Q, Zhang Y, Cheng Z, Chen M, Wang S, Feng Q, Huang Y, Li Y, Tang Y, Zhou B, et al: A fine physical map of the rice chromosome 4. Genome Res 2002, 12(5):817-823.

19. Kim JS, Islam-Faridi MN, Klein PE, Stelly DM, Price HJ, Klein RR, Mullet JE: Comprehensive molecular cytogenetic analysis of sorghum genome architecture: distribution of euchromatin, heterochromatin, genes and recombination in comparison to rice. Genetics 2005, 171(4):1963-1976.

20. Paterson AH, Bowers JE, Bruggmann R, Dubchak I, Grimwood J, Gundlach H, Haberer G, Hellsten U, Mitros T, Poliakov A, et al: The Sorghum bicolor genome and the diversification of grasses. Nature 2009, 457(7229):551-556.

21. Hyten DL, Choi I, Song Q, Specht JE, Carter TE, Shoemaker RC, Hwang EY, Matukumalli LK, Cregan PB: A high density integrated genetic linkage map of soybean and the development of a 1536 universal soy linkage panel for quantitative trait locus mapping. Crop Sci 2010, 50(3):960-968

22. Brummer EC, Graef GL, Orf J, Wilcox JR, Shoemaker RC: Mapping QTL for seed protein and oil content in eight soybean populations. Crop Sci 1997, 37(2):370-378

23. Specht JE, Chase K, Macrander M, Graef GL, Chung J, Markwell JP, Germann M, Orf JH, Lark KG: Soybean response to water: a QTL analysis of drought tolerance. Crop Sci 2001, 41(2):493-509.

24. Mansur LM, Orf JH, Chase K, Jarvik T, Cregan PB, Lark KG: Genetic mapping of agronomic traits using recombinant inbred lines of soybean. Crop Sci 1996, 36(5):1327-1336.

25. Lee SH, Bailey MA, Mian MAR, Carter TE Jr, Shipe ER, Ashley DA, Parrott WA Hussey RS, Boerma HR: RFLP loci associated with soybean seed protein and oil content across populations and locations. Theor App/ Genet 1996, 93(5/6):649-657

26. Orf JH, Chase K, Jarvik T, Mansur LM, Cregan PB, Adler FR, Lark KG: Genetics of soybean agronomic traits: I: comparison of three related recombinant inbred populations. Crop Sci 1999, 39(6):1642-1651.

27. Stombaugh SK, Orf JH, Jung HG, Chase K, Lark KG, Somers DA: Quantitative trait loci associated with cell wall polysaccharides in soybean seed. Crop Sci 2004, 44:2101-2106.

28. Raychaudhuri S: Mapping rare and common causal alleles for complex human diseases. Cell 2011, 147(1):57-69.

29. Ladouceur M, Dastani Z, Aulchenko YS, Greenwood CM, Richards JB: The empirical power of rare variant association methods: results from sanger sequencing in 1,998 individuals. PLoS Genet 2012, 8(2):e1002496.

30. Chung J, Babka HL, Graef GL, Staswick PE, Lee DJ, Cregan PB, Shoemaker $\mathrm{RC}$, Specht JE: The seed protein, oil, and yield QTL on soybean linkage group I. Crop Sci 2003, 43(3):1053-1067.

31. Diers BW, Keim P, Fehr WR, Shoemaker RC: RFLP analysis of soybean seed protein and oil content. Theor Appl Genet 1992, 83(5):608-612.

32. Nichols DM, Glover KD, Carlson SR, Specht JE, Diers BW: Fine mapping of a seed protein QTL on soybean linkage group I and its correlated effects on agronomic traits. Crop Sci 2006, 46:834-839.

33. Reinprecht Y, Poysa WW, Yu K, Rajcan I, Ablett GR, Pauls KP: Seed and agronomic QTL in low linolenic acid, lipoxygenase-free soybean (Glycine max (L.) Merrill) germplasm. Genome 2006, 49(12):1510-1527.

34. Sebolt AM, Shoemaker RC, Diers BW: Analysis of a quantitative trait locus allele from wild soybean that increases seed protein concentration in soybean. Crop Sci 2000, 40(5):1438-1444.

35. Tajuddin T, Watanabe S, Yamanaka N, Harada K: Analysis of quantitative trait loci for protein and lipid contents in soybean seeds using recombinant inbred lines. Breeding Science 2003, 53:133-140.

36. Bolon YT, Joseph B, Cannon SB, Graham MA, Diers BW, Farmer AD, May GD, Muehlbauer GJ, Specht JE, Tu ZJ, et al: Complementary genetic and genomic approaches help characterize the linkage group I seed protein QTL in soybean. BMC Plant Biol 2010, 10:41

37. Cardon LR, Bell Jl: Association study designs for complex diseases. Nat Rev Genet 2001, 2(2):91-99.

38. Brim CA, Burton JW: Recurrent selection in soybeans: II: selection for increased percent protein in seeds. Crop Sci 1979, 19:494-498.

39. Cober ER, Voldeng HD: Developing high-protein, high-yield soybean populations and lines. Crop Sci 2000, 40:39-42.

40. Wilcox JR: Increasing seed protein in soybean with eight cycles of recurrent selection. Crop Sci 1998, 38(6):1536-1540. 
41. Burton JW: Quantitative genetics: results relevant to soybean breeding. In Soybeans: Improvement, production, and Uses. 2nd edition. Edited by Wilcox JR. 1987:211-247.

42. Bao XU: A decade of wild soybean (Glycine soja) research in China. Jilin Agric Sci 1989, 39(1):5-13.

43. Xu B: The protein resources of Glycine max in China. Soybean Sci 1985, 3(4):327-331.

44. Wilson RF: Seed composition: p. 621-677. In HR Boerma and JE Specht (ed) Soybean: Improvement, production, and Uses 3rd ed. 2004.

45. Song Q, Hyten DL, Jia G, Quigley CV, Fickus EW, Nelson RL, Cregan PB: Development and evaluation of SoySNP50K, a high-density genotyping array for soybean. PLoS One 2013, 8(1):e54985.

46. Keim P, Olson TC, Shoemaker RC: A rapid protocol for isolating soybean DNA. Soybean Genet Newsl 1988, 15:150-152.

47. Campbell CR: Total carbon, hydrogen and nitrogen-combustion method. Res Ser Bull 1992, 368:21-23.

48. Rubel G: Simultaneous determination of oil and water contents in different oilseeds by pulsed nuclear magnetic resonance. J Am Oil Chem Soc 1994, 71(10):1057-1062.

49. Fan JB, Gunderson KL, Bibikova M, Yeakley JM, Chen J, Wickham Garcia E, Lebruska LL, Laurent M, Shen R, Barker D: Illumina universal bead arrays. Methods Enzymol 2006, 410:57-73.

50. Hyten DL, Song Q, Choi I, Yoon M, Specht JE, Matukumalli LK, Nelson RL, Shoemaker RC, Young ND, Cregan PB: High-throughput genotyping with the GoldenGate assay in the complex genome of soybean. Theor Appl Genet 2008, 116(7):945-952.

51. Choi IY, Hyten DL, Matukumalli LK, Song Q, Chaky JM, Quigley CV, Chase K, Lark KG, Reiter RS, Yoon MS, et al: A soybean transcript map: gene distribution, haplotype and single-nucleotide polymorphism analysis. Genetics 2007, 176:685-696.

52. Barrett JC, Fry B, Maller J, Daly MJ: Haploview: analysis and visualization of LD and haplotype maps. Bioinformatics 2005, 21(2):263-265.

53. Gaut BS, Long AD: The lowdown on linkage disequilibrium. Plant Cell 2003, 15(7):1502-1506.

54. Cregan PB, Jarvik T, Bush AL, Shoemaker RC, Lark KG, Kahler AL, Kaya N, VanToai TT, Lohnes DG, Chung J: An integrated genetic linkage map of the soybean genome. Crop Sci 1999, 39(5):1464-1490.

55. Song QJ, Marek LF, Shoemaker RC, Lark KG, Concibido VC, Delannay X, Specht JE, Cregan PB: A new integrated genetic linkage map of the soybean. Theor Appl Genet 2004, 109(1):122-128.

56. Zhu YL, Song QJ, Hyten DL, Van Tassell CP, Matukumalli LK, Grimm DR, Hyatt SM, Fickus EW, Young ND, Cregan PB: Single-nucleotide polymorphisms in soybean. Genetics 2003, 163(3):1123-1134.

57. Song Q, Jia G, Zhu Y, Grant D, Nelson RT, Hwang EY, Hyten DL, Cregan P: Abundance of SSR motifs and development of candidate polymorphic SSR markers (BARCSOYSSR_1.0) in soybean. Crop Sci 2010, 50(5):1950-1960.

58. Alexander DH, Novembre J, Lange K: Fast model-based estimation of ancestry in unrelated individuals. Genome Res 2009, 19(9):1655-1664.

59. Yu J, Pressoir G, Briggs WH, Vroh Bi I, Yamasaki M, Doebley JF, McMullen MD, Gaut BS, Nielsen DM, Holland JB, et al: A unified mixed-model method for association mapping that accounts for multiple levels of relatedness. Nat Genet 2006, 38(2):203-208.

doi:10.1186/1471-2164-15-1

Cite this article as: Hwang et al:: A genome-wide association study of seed protein and oil content in soybean. BMC Genomics 2014 15:1.

\section{Submit your next manuscript to BioMed Central and take full advantage of:}

- Convenient online submission

- Thorough peer review

- No space constraints or color figure charges

- Immediate publication on acceptance

- Inclusion in PubMed, CAS, Scopus and Google Scholar

- Research which is freely available for redistribution

Submit your manuscript at www.biomedcentral.com/submit 